Article

\title{
Chemical Composition and Antioxidant Activity of Thyme, Hemp and Coriander Extracts: A Comparison Study of Maceration, Soxhlet, UAE and RSLDE Techniques
}

\author{
Sara Palmieri, Marika Pellegrini $\left.{ }^{+} \mathbb{(}\right)$, Antonella Ricci * ${ }^{\mathbb{C}}$, Dario Compagnone $\mathbb{}$ \\ and Claudio Lo Sterzo \\ Faculty of Bioscience and Technology for Food, Agriculture and Environment, University of Teramo, \\ Via R. Balzarini 1, 64100 Teramo, Italy; spalmieri@unite.it (S.P.); mpellegrini@unite.it (M.P.); \\ dcompagnone@unite.it (D.C.); closterzo@unite.it (C.L.S.) \\ * Correspondence: aricci@unite.it; Tel.: +39-0861-266-904 \\ † Present address: Department of Life, Health and Environmental Sciences, University of L'Aquila, Via Vetoio 9 , \\ 67010 Coppito, L'Aquila, Italy.
}

Received: 1 August 2020; Accepted: 27 August 2020; Published: 2 September 2020

check for updates

\begin{abstract}
Appropriate and standardized techniques for the extraction of secondary metabolites with interesting biological activity from plants are required. In this work, a comparison of different conventional and unconventional extraction techniques (maceration-M, Soxhlet- $S$, ultrasound assisted extraction-UAE, and rapid solid-liquid dynamic extraction-RSLDE) was investigated. Bioactive compounds were extracted from Thymus vulgaris L. (thyme), Cannabis sativa L. (industrial hemp) and Coriandrum sativum L. (coriander) and chemically characterized for their volatile fraction and polyphenolic content by means of gas chromatography-mass spectrometry (GC-MS) and high performance liquid chromatography-ultraviolet (HPLC-UV). Linalool (48.19\%, RSLDE) and carvacrol $(21.30 \%, \mathrm{M})$ for thyme, caryophyllene $(54.78 \%, \mathrm{~S})$ and humulene $(14.13 \%, \mathrm{~S})$ for hemp, and linalool $(84.16 \%$, RSLDE) for coriander seeds were the main compounds among terpenes, while thyme was the richest source of polyphenols with rosmarinic acid (51.7 mg/g dry extract-S), apigenin (7.6 mg/g dry extract-S), and luteolin ( $4.1 \mathrm{mg} / \mathrm{g}$ dry extract-UAE) being the most abundant. In order to shed light on their potential as natural food preservatives, the biological activity of the extracts was assessed in terms of antioxidant activity (2,2' -azino-bis(3-ethylbenzothiazoline-6-sulphonic acid-ABTS ${ }^{+}$, ferric reducing antioxidant power-FRAP, 2,2-diphenyl-1-picrylhydrazyl-DPPH assays) and phenolic content (Folin-Ciocâlteu method). For thyme, Soxhlet extracts showed best performances in FRAP and ABTS ${ }^{+}$assays $(74 \mathrm{mg}$ TE/g dry extract and $134 \mathrm{mg}$ TE/g dry extract, respectively), while Soxhlet and RSLDE extracts recorded similar activity in DPPH' (107-109 mg TE/g dry extract). For hemp and coriander, indeed, RSLDE extracts accounted for higher antioxidant activity as evidenced by FRAP ( $80 \mathrm{mg}$ TE/g dry extract and $18 \mathrm{mg}$ TE/g dry extract, respectively) and $\mathrm{ABTS}^{+}$(557 mg TE/g dry extract and $48 \mathrm{mg}$ TE/g dry extract, respectively) assays. With respect to $\mathrm{DPPH}$; the best results were observed for UAE extracts (45 mg TE/g dry extract and $220 \mathrm{mg}$ $\mathrm{TE} / \mathrm{g}$ dry extract, respectively). Our findings suggest that all the investigated techniques are valid extraction methods to retain bioactive compounds and preserve their activity for application in food and pharmaceutical formulations. Among them, the innovative RSLDE stands out for the slightly higher antioxidant performances of the extracts, coupled with the facility of use and standardization of the extraction process.
\end{abstract}

Keywords: ultrasound assisted extraction-UAE; rapid solid-liquid dynamic extraction-RSLDE; gas chromatography-mass spectrometry-GC-MS; antioxidants; C. sativa; T. vulgaris; C. sativum 


\section{Introduction}

Plant bioactive compounds are defined as secondary plant metabolites capable of exerting a positive effect on animal or human health. Secondary metabolites are produced within the plants beyond the primary biosynthetic and metabolic routes of compounds [1]. These components are not needed for plant basic metabolism, can be regarded as products of biochemical "sidetracks" in the plant cells, and can cover important functions in living plants. Polyphenols, for example, can protect plants against free radicals generated during photosynthesis. Terpenoids may attract pollinators or seed dispersers or inhibit competing plants, whereas alkaloids usually ward off herbivore animals or insect attacks.

Among the best-known bioactive compounds, polyphenols and terpenes can delay or inhibit the oxidation of lipids or other biomolecules, and, thus, prevent or repair the damage of human cells caused by oxygen $[2,3]$. The importance of these components has been emphasized in the last years. The ever-increasing consumer sensibility to the consumption of food with lower content of synthetic chemical products and the loss of efficacy of common preservatives, due to the development and diffusion of resistant bacteria, have led to increasing research activities regarding the extraction and the evaluation of the efficacy of natural antioxidants $[4,5]$.

The use of plant bioactive compounds as antioxidants in different commercial sectors, such as the pharmaceutical, food, and chemical industries, needs an appropriate and standardized extraction technique [6]. Extraction is the first step of any plant chemical component study and plays a significant and crucial role. The efficiency of conventional and non-conventional extraction methods strongly depends on the input parameters, the nature of the plant matrix, the chemistry of bioactive compounds, and the operator expertise $[7,8]$.

Traditional methods, like maceration, percolation, and Soxhlet, are known to have some limits such as time and solvent consumption, and decomposition of heat sensitivity bioactive compounds [8]. However, Soxhlet technique is still common in laboratories and industries being involved in a wide variety of official methods [9]. Recently, the need of enhancing the biological activity of plant extracts has led to the development of unconventional extraction methods. Among the latter, microwave assisted extraction (MAE), supercritical fluid extraction (SFE), ultrasound assisted extraction (UAE), and rapid solid-liquid dynamic extraction (RSLDE) are the most interesting [10-12].

In UAE, the propagation of ultrasonic waves through a liquid medium damages plant wall, resulting in an improvement in solvent penetration; thus, bioactive components can be extracted in minutes. Therefore, with respect to conventional methods, UAE has the advantage of reducing the extraction process time and energy consumption retaining high efficiency $[13,14]$.

The RSLDE, performed by Naviglio Extractor ${ }^{\circledR}$, can be considered among the "greenest" strategies, operating at room temperature, with a minimum waste of energy and solvents. Naviglio's principle is based on generating, with a suitable solvent, a negative pressure gradient between the internal and external sides of a solid matrix containing extractable material, followed by a sudden restoration of the initial equilibrium conditions. This process induces the forced extraction of the compounds not chemically linked to the main structure of the solid [15].

Scientific literature presents several works about RSLDE comparison with other extraction techniques. However, few records of this comparison are aimed at food preservation [16-19]. The present work focuses on the comparison of different conventional and unconventional extraction techniques (maceration, Soxhlet, UAE, and RSLDE), to obtain extracts suitable for food preservation. Three aromatic species were investigated: Thymus vulgaris L., Cannabis sativa L., and Coriandrum sativum L. The obtained extracts were chemically characterized, and their biological activity was assessed in terms of antioxidant activity. 


\section{Materials and Methods}

\subsection{Plant Material}

Plants were open field cultivated in Abruzzo's territory starting from certified seeds. Dry inflorescences of Cannabis sativa 'Futura 75' (hemp), dry apical stems and leaves of Thymus vulgaris (thyme) and seeds of Coriandrum sativum (coriander) were obtained from a local farmer (Hemp Farm Italia, Tortoreto (TE), Azienda Agricola Luigi Barlafante, Roseto degli Abruzzi (TE), and Mediterranea Sementi, Sant'Atto (TE), respectively).

Inflorescences of hemp were collected during the flowering period (September), let dry in a dark room at room temperature $\left(20-25^{\circ} \mathrm{C}\right)$, with controlled relative humidity $(45-55 \%)$, and stored in the same conditions until processing. Little branches of T. vulgaris were collected during the balsamic period (June), dried on the field, and stored in a dry and darkroom until processing. Seed heads of C. sativum were cut off when the plant began to turn brown, put in a paper bag, and hanged. After drying, seeds were collected and stored in sealed bags.

\subsection{Chemicals}

Ethanol absolute was obtained from Carlo Erba (Milan, Italy). Acetic acid, acetonitrile, methanol, and water (high performance liquid chromatography-HPLC grade) were purchased from VWR (Milan, Italy).

$\alpha$-pinene, $\beta$-pinene, linalool, $\beta$-myrcene, terpinolene, caryophyllene, humulene, and $\beta$-bisabolene, gallic acid, $p$-OH benzoic acid, chlorogenic acid, vanillic acid, caffeic acid, syringic acid, ferulic acid, and rosmarinic acid (from Sigma-Aldrich, Darmstadt, Germany) standards were employed. Working standard mixtures were prepared by appropriate dilution of the standards in methanol. All solutions were stored at $-20{ }^{\circ} \mathrm{C}$ in the dark.

Folin-Ciocâlteu's reagent, 6-hydroxy-2,5,7,8-tetramethylchroman-2-carboxylic acid (Trolox), 2,2-diphenyl-1-picrylhydrazyl (DPPH), and 2,2'-azino-bis(3-ethylbenzothiazoline-6-sulphonic acid) diammonium salt $\left(\mathrm{ABTS}^{+}\right)$were purchased from Sigma-Aldrich (Darmstadt, Germany). Sodium carbonate, potassium persulfate, potassium hexacyanoferrate(III), trichloroacetic acid, ferric chloride, and potassium phosphate monobasic were obtained from Carlo Erba (Milan, Italy).

\subsection{Extractions}

Before extraction, the samples were homogenized by trituration with a chopper (Kenwood Quad Blade CH580 Chopper, Kenwood Limited, Havant, UK) 3 times and then crushed with a mortar. Trituration time was as follows: hemp inflorescences, $10 \mathrm{~s}$; thyme leaves and little stems, $20 \mathrm{~s}$; coriander seeds, $15 \mathrm{~s}$.

Extracts were obtained both with conventional methods, as maceration and Soxhlet, and using the unconventional UAE and RSLDE. RSLDE and Soxhlet extracts were produced with two commonly utilized total time extraction processes: 2 and $6 \mathrm{~h}$.

The extracts were all collected in flasks, filtered, and brought to dry by Rotavapor Steroglass S.r.l. (Perugia, Italy).

The extraction yields were calculated according to the equation:

$$
\text { Yield }(\% w / w)=\frac{\text { mass dried extract }(g)}{\text { mass dried matrix }(g)} \times 100
$$

The results were expressed as the average of two replicates of the extraction.

\subsubsection{RSLDE Extraction}

RSLDE technique was performed using Naviglio Extractor ${ }^{\circledR}$ (Atlas Filtri, Padua, Italy), using the same quantitative for both extraction processes (at 2 and $6 \mathrm{~h}$ ): $50 \mathrm{~g}$ of inflorescences for C. sativa, $20 \mathrm{~g}$ of leaves and stems for T. vulgaris and $106 \mathrm{~g}$ of seeds for C. sativum. $250 \mathrm{~mL}$ of ethanol were used as 
extraction solvent. The 2 hours process (N2h) was carried out by processing plant matrix for 30 cycles (with a maximum pressure of 8 bar); each cycle was composed by 12 hits in the dynamic phase ( 2 min duration) and a duration of the static phase of $2 \mathrm{~min}$. The $6 \mathrm{~h}$ extracts (N6h) were obtained with the same conditions, but with a major number of cycles (i.e., 90 ).

\subsubsection{Soxhlet Extraction}

Soxhlet extracts were produced starting from the same quantitative for both extraction processes (at 2 and $6 \mathrm{~h}$, S2h and S6h, respectively): $50 \mathrm{~g}$ of inflorescences for C. sativa, $20 \mathrm{~g}$ of leaves and stems for T. vulgaris and $106 \mathrm{~g}$ of seeds for $C$. sativum were used. The extractions were performed with $250 \mathrm{~mL}$ of ethanol at $100{ }^{\circ} \mathrm{C}$.

\subsubsection{Maceration}

Macerations were performed using $9 \mathrm{~g}$ of inflorescences for C. sativa, $4 \mathrm{~g}$ of leaves and stems for T. vulgaris, and $21 \mathrm{~g}$ of seeds for C. sativum. The macerates (M) were obtained with $50 \mathrm{~mL}$ of ethanol as solvent for 30 days at room temperature without light exposure.

\subsubsection{UAE Extraction}

The UAE extractions were performed using $19 \mathrm{~g}$ of inflorescences for $C$. sativa, $8 \mathrm{~g}$ of leaves and little stems for T. vulgaris and $42 \mathrm{~g}$ of seeds for C. sativum. Plant matrices were extracted with $100 \mathrm{~mL}$ of ethanol in $250 \mathrm{~mL}$ flasks, sealed and immersed in an ultrasonic water bath (Argo Lab DU-45, Milan, Italy) for $15 \mathrm{~min}(40 \mathrm{kHz}, 180 \mathrm{~W})$.

\subsection{SPME/GC-MS Characterization of Extracts Volatile Fraction}

Chemical characterizations of extracts volatile fraction were performed by solid-phase microextraction/gas chromatography coupled to mass spectrometry (SPME/GC-MS). SPMEs were obtained by a Supelco-57299-U SPME DVB/CAR/PDMS (Divinylbenzene/Carboxen/Polydimethylsiloxane) fiber (Sigma Aldrich-Saint Louis, MO, USA). All extracts were processed as follows: $0.50 \mathrm{~g}$ of dry extract were put into a $20 \mathrm{~mL}$ capacity glass vial and sealed with a rubber septum and an aluminum. The vial was placed on a heated plate $\left(50^{\circ} \mathrm{C}\right)$ and the SPME needle was inserted into the vial. The grey fiber was exposed to the headspace for $20 \mathrm{~min}$. After exposure, the fiber was retracted into a needle and loaded into the injection port of the gas chromatographer for fiber desorption at $250{ }^{\circ} \mathrm{C}$ for $15 \mathrm{~min}$.

A Clarus 580 GC apparatus (PerkinElmer-Waltham, MA, USA) coupled to a Clarus SQ 8 S GC/MS (PerkinElmer-Waltham, MA, USA) was used for GC-MS analysis. Separations were achieved on a fused silica Zebron-ZB-SemiVolatile column (30 m × $250 \mu \mathrm{m} \times 0.25 \mu \mathrm{m}$-Phenomenex, Torrance, CA, USA). Analyses were carried following a different temperature gradient depending on samples.

The temperature gradient for hemp extracts was as follows: starting temperature $50^{\circ} \mathrm{C}$ (hold $1 \mathrm{~min}$ ), up to $145^{\circ} \mathrm{C}$ at $7{ }^{\circ} \mathrm{C} / \mathrm{min}$ (hold $5 \mathrm{~min}$ ), up to $175^{\circ} \mathrm{C}$ at $4{ }^{\circ} \mathrm{C} / \mathrm{min}$ and up to $250{ }^{\circ} \mathrm{C}$ at $7{ }^{\circ} \mathrm{C} / \mathrm{min}$ (hold $5 \mathrm{~min}$ ). The carrier gas was Helium (flow rate $1 \mathrm{~mL} / \mathrm{min}$ ). The split of the injector was set to 1:50, while the injector and the transfer line temperature were set at $250{ }^{\circ} \mathrm{C}$.

The temperature gradient for thyme and coriander extracts was as follows: starting temperature $45^{\circ} \mathrm{C}$ (hold $10 \mathrm{~min}$ ), up to $180{ }^{\circ} \mathrm{C}$ at $2.5^{\circ} \mathrm{C} / \mathrm{min}$ (hold $5 \mathrm{~min}$ ). The carrier gas was Helium (flow $1 \mathrm{~mL} / \mathrm{min}$ ), while the injector and the transfer line temperature were set at $250{ }^{\circ} \mathrm{C}$.

The semi-quantitative characterization was carried out through Turbomass 6.1.0.1963 software (PerkinElmer-Waltham, MA, USA). The unknown compounds were identified by matching the obtained spectra with the NIST Mass Spectral Library 2.0 (NIST-Gaithersburg, MD, USA) and confirmed by comparison of the retention index (RI) with those retrieved from http://webbook.nist.gov/chemistry/. A mix of $n$-alkanes, ranging from octane (C8) to triacontane (C30) was obtained from Supelco (Bellefonte, CA, USA) and injected using the analytical conditions above reported to determine the retention index (RI) as proposed by Lee et al. [20]. 
Semi-quantitative analysis was made by peak area normalization without response factors. Relative abundances (\%) were the mean of two replicates.

\subsection{HPLC-UV Characterization of the Phenolic Fraction}

Phenolic compounds were determined by HPLC (Perkin-Elmer series 200, Monza, Italy) equipped with an autosampler and a UV-Vis detector (Perkin Elmer LC 240, Monza, Italy) set at $280 \mathrm{~nm}$. For separation, a Phenomenex Kinetex C18 column was used (dimensions: $250 \times 4.6 \mathrm{~mm}$, particle size: $5 \mu \mathrm{m}$, pore size: $110 \AA$; Phenomenex, Bologna, Italy). The mobile phases used were: (A) $1 \%$ acetic acid in water and (B) acetonitrile. For analyte separation, the mobile phase gradient was programmed as follows: from $10 \%$ to $100 \%$ solvent $\mathrm{B}$ for $30 \mathrm{~min}$ and subsequent return to initial composition in $4 \mathrm{~min}$, achieving mobile phase stabilization for $10 \mathrm{~min}$.

$40 \mathrm{mg}$ of dry extracts sample was dissolved in $1 \mathrm{~mL}$ of water/methanol (50:50), vortexed for $3 \mathrm{~min}$, centrifugated for $15 \mathrm{~min}$, filtered with $0.2 \mu \mathrm{m}$ PTFE filter and analyzed.

Quantification of polyphenols was carried out by the external standard method. Linear regression curves based on peak area were calculated for each phenolics compound after injection of mix phenolic standard solutions covering the sample range of concentrations (6-12-25-50-100 ppm).

For quantitative analysis, a calibration curve for each available phenolic standard were constructed based on the UV signal: gallic acid $\left(y=36,255 x-26,062 ; R^{2}=0.9983\right), p-O H-b e n z o i c(y=31,711 x-$ $\left.16,966) ; R^{2}=0.9992\right)$, vanillic acid $\left(y=33,123 x-52,417 ; R^{2}=0.9974\right)$, rosmarinic acid $(y=34,344 x-$ 40,$\left.066 ; R^{2}=0.9992\right)$, ferulic acid $\left(y=61,245 x+74,735 ; R^{2}=0.9912\right)$, caffeic acid $(y=44,841 x-416,813$; $0.9952)$, syringic acid $\left(y=39,490 x-106,101 ; R^{2}=0.9987\right)$, luteolin $\left(y=7593,1 x-19,075 ; R^{2}=0.9991\right)$, apigenin $\left(y=83,755 x-7443,3 ; R^{2}=0.9979\right)$, and chlorogenic acid $\left(y=29,136 x-63,864 ; R^{2}=0.9971\right)$.

\subsection{Total Phenolic Content (TPC) and Antioxidant Capacity (AOC)}

Total Phenolic Content (TPC) estimation was carried out by means of Folin-Ciocâlteu's reagent, following the Singelton and Rossi method [21]. The reference standard was gallic acid (GA). Results are expressed as mg GA equivalents (GAE)/g dry extract, mean value of two replicates.

The antioxidant activity (AOC) was investigated employing:

- DPPH assay, following the method proposed Brand-Williams et al. [22];

- $\mathrm{ABTS}^{+}$assay, with the Gullon et al. method [23],

- FRAP assay, assessed by means of potassium ferricyanide-ferric chloride method described by Oyaizu [24].

For FRAP, DPPH; and ABTS ${ }^{+}$assays, Trolox was used as a reference standard. Results are expressed as mg Trolox equivalents (TE)/g dry extract, mean value of two replicates.

\subsection{Statistical Analysis}

Results were expressed as means \pm standard deviations. Yields, chemical, and biological characterization data were subjected to ANOVA (analysis of variance), followed by Tukey's HSD post-hoc test at a significance level of $5 \%(p<0.05)$. Terpenes classes composition obtained by SPME/GC-MS were processed through principal component analysis (PCA) to observe the possible correlations within the extracts of the different matrices. Before applying the PCA algorithm, the data were linearized and automatically scaled (zero mean and unit variance) to eliminate the differences in the concentration range. The data set consisted of $18 \times 4$, in which rows represented the 18 extracts and columns the 4 terpenes classes. Data on terpenes classes were also treated using a hierarchical clustering method. Dendrograms were constructed using Euclidean distance measure and Ward's method of dissimilarity between clusters. Both statistical tests were performed with Microsoft Xlstat 2016 statistical software (Addinsoft, Paris, France). 


\section{Results and Discussion}

\subsection{Yields}

The extracts yields obtained for the three plant matrices are reported in Table 1. The highest yield for thyme was obtained for $2 \mathrm{~h}$ Soxhlet extraction (S2h), while the lowest for maceration (M) and ultrasound assisted extraction (UAE) $(p<0.05)$. The best yield for hemp was achieved, indeed, for $6 \mathrm{~h}$ Soxhlet extraction (S6h), while the lowest for M and UAE extracts $(p<0.05)$. For this matrix, no significant differences were recorded among RSLDE extraction times (N2h and N6h) and S2h ( $p>0.05)$. A totally different behavior was observed for coriander seeds extracts: the best yield was found for $\mathrm{UAE}$ and, then M; the lowest was N2h.

According to these data, Soxhlet seems to be the most suitable technique for the extraction of plants aerial parts in terms of yield. However, it should be pointed out that this extraction technique carried out at high temperature allows the co-extraction of the fibers [25-29]. These contribute to the dry extract weight. On the other hand, ultrasound assisted method seems to be the best extraction technique to process plant seeds.

Lower yields were obtained for coriander seeds with respect to hemp and thyme. This is common to other plant species. In fact, the best yields of extraction are usually recovered from stems and leaves $[30,31]$. In any case, our findings are in line with literature data, falling within the intervals normally reported in several works for the same species for some of these techniques [28,32].

Table 1. Yields of extracts $(\% w / w)$. N2h, RSLDE 2 h; N6h, RSLDE 6 h; S2h, Soxhlet 2 h; S6h, Soxhlet 6 h; $\mathrm{UAE}$, ultrasound assisted extraction; $\mathrm{M}$, maceration.

\begin{tabular}{ccccccc}
\hline & N2h & N6h & S2h & S6h & UAE & M \\
\hline Thyme & $2.30 \pm 0.06 \mathrm{~d}$ & $2.45 \pm 0.09 \mathrm{c}$ & $9.25 \pm 0.02 \mathrm{a}$ & $8.65 \pm 0.05 \mathrm{~b}$ & $1.62 \pm 0.02 \mathrm{e}$ & $1.78 \pm 0.04 \mathrm{e}$ \\
Hemp & $6.00 \pm 0.03 \mathrm{~b}$ & $5.81 \pm 0.05 \mathrm{~b}$ & $5.70 \pm 0.01 \mathrm{~b}$ & $10.00 \pm 0.07 \mathrm{a}$ & $0.71 \pm 0.09 \mathrm{c}$ & $0.95 \pm 0.04 \mathrm{c}$ \\
Coriander seeds & $0.57 \pm 0.09 \mathrm{f}$ & $0.73 \pm 0.08 \mathrm{e}$ & $1.18 \pm 0.06 \mathrm{~d}$ & $1.63 \pm 0.09 \mathrm{c}$ & $2.36 \pm 0.07 \mathrm{a}$ & $2.17 \pm 0.08 \mathrm{~b}$ \\
\hline
\end{tabular}

Results followed by the same case-letter are not different according to Tukey's HSD post-hoc test ( $p>0.05)$.

\subsection{Chemical Composition of Extracts Volatile Fraction}

The SPME/GC-MS characterization data of the volatile fraction of the extracts of the three plants are shown in Table 2.

In thyme extracts 22 compounds were found, 21 monoterpenes, and one sesquiterpene. Thymol has generally been reported to be the main component of T. vulgaris. However, this cultivar contains carvacrol, the isomer of thymol, that has the same biological activity. Linalool was the most abundant volatile compound in all the extracts, but using Soxhlet for two hours, a much lower quantity was found. Both carvacrol and linalool are natural effective antimicrobials used to control the growth of spoilage microorganisms in food as demonstrated in some studies in literature [33,34]. They have been reported to have also therapeutic properties (e.g., vs. Alzheimer's disease) [35].

A total of 25 compounds were identified in hemp extracts, 13 monoterpenes, and 12 sesquiterpenes. The predominant compounds were: $\beta$-myrcene and caryophyllene within the monoterpenes and sesquiterpenes, respectively. $\beta$-myrcene is known to possess anti-inflammatory, analgesic, and anxiolytic properties $[36,37]$. Caryophyllene has been reported as anti-inflammatory compound in some cannabis preparations because of the interaction with the cannabinoid receptors and a gastric cytoprotective activity has been also found [38-40]. Interestingly, caryophyllene oxide seems to be a multi-target molecule, known for its anticancer and analgesic properties [37]. 
Table 2. Solid-phase microextraction/gas chromatography-mass spectrometry (SPME/GC-MS) characterization of the volatile fraction of thyme, hemp and coriander seeds extracts. Results are expressed as relative abundances \% (means $\pm \mathrm{sd}$ ).

\begin{tabular}{|c|c|c|c|c|c|c|c|c|}
\hline ID-Thyme & Terpenes Class & RI & N2h & N6h & $\mathrm{S} 2 \mathrm{~h}$ & S6h & UAE & $\mathbf{M}$ \\
\hline$\alpha$-pinene & bicyclic monoterpenes & 921 & $2.11 \pm 0.73$ & $2.34 \pm 0.31$ & $2.53 \pm 0.19$ & $2.26 \pm 0.10$ & $2.47 \pm 0.35$ & $1.57 \pm 0.16$ \\
\hline sabinene & bicyclic monoterpenes & 967 & $2.49 \pm 0.61$ & $2.82 \pm 0.27$ & $2.36 \pm 0.09$ & $2.90 \pm 0.22$ & $3.04 \pm 0.22$ & $2.21 \pm 0.25$ \\
\hline 1-octen-3-ol & acyclic monoterpenes & 980 & $0.63 \pm 0.02$ & $0.31 \pm 0.01$ & $0.30 \pm 0.00$ & $0.61 \pm 0.04$ & $0.82 \pm 0.13$ & $0.22 \pm 0.00$ \\
\hline$\beta$-myrcene & acyclic monoterpenes & 999 & $0.52 \pm 0.02$ & $0.56 \pm 0.08$ & $0.82 \pm 0.23$ & $0.60 \pm 0.01$ & $0.56 \pm 0.03$ & $0.43 \pm 0.00$ \\
\hline X-3-carene & bicyclic monoterpenes & 1010 & $3.52 \pm 0.38$ & $4.20 \pm 0.94$ & $3.26 \pm 0.96$ & $4.16 \pm 0.04$ & $4.10 \pm 0.19$ & $3.15 \pm 0.15$ \\
\hline$o$-cymene & monocyclic monoterpenes & 1019 & $0.18 \pm 0.01$ & $0.90 \pm 0.08$ & $1.24 \pm 0.54$ & $0.45 \pm 0.08$ & $1.31 \pm 0.31$ & $0.16 \pm 0.01$ \\
\hline p-cymene & monocyclic monoterpenes & 1023 & $1.18 \pm 0.04$ & $1.33 \pm 0.20$ & $7.81 \pm 0.26$ & $1.38 \pm 0.10$ & $1.34 \pm 0.03$ & $1.01 \pm 0.04$ \\
\hline V-terpinene & monocyclic monoterpenes & 1054 & $5.45 \pm 0.49$ & $3.21 \pm 0.54$ & $5.48 \pm 0.16$ & $7.17 \pm 0.80$ & $6.81 \pm 0.19$ & $5.03 \pm 0.22$ \\
\hline cis-sabinene hydrate & bicyclic monoterpenes & 1066 & $5.93 \pm 0.76$ & $4.88 \pm 0.26$ & $2.29 \pm 0.53$ & $5.06 \pm 0.42$ & $5.96 \pm 0.05$ & $4.14 \pm 0.09$ \\
\hline cis-linalool oxide & monocyclic monoterpenes & 1079 & $1.14 \pm 0.14$ & $1.30 \pm 0.27$ & $5.68 \pm 0.33$ & $1.33 \pm 0.06$ & $1.20 \pm 0.04$ & $1.03 \pm 0.01$ \\
\hline linalool & acyclic monoterpenes & 1096 & $48.19 \pm 1.95$ & $46.32 \pm 1.23$ & $16.19 \pm 2.88$ & $42.59 \pm 1.37$ & $47.66 \pm 3.79$ & $44.95 \pm 1.81$ \\
\hline cis-p-menth-2-en-1-ol & monocyclic monoterpenes & 1123 & $2.03 \pm 0.65$ & $0.81 \pm 0.02$ & $14.36 \pm 0.19$ & $0.37 \pm 0.05$ & $0.68 \pm 0.01$ & $0.19 \pm 0.01$ \\
\hline trans-limonene oxide & monocyclic monoterpenes & 1138 & $2.20 \pm 0.54$ & $0.89 \pm 0.11$ & $1.24 \pm 1.68$ & $0.15 \pm 0.02$ & $0.19 \pm 0.07$ & $0.40 \pm 0.00$ \\
\hline$\beta$-pinene oxide & bicyclic monoterpenes & 1155 & $0.01 \pm 0.00$ & $1.06 \pm 0.01$ & $6.92 \pm 0.86$ & $0.36 \pm 0.04$ & $0.79 \pm 0.02$ & $0.30 \pm 0.00$ \\
\hline trans-linalool oxide & monocyclic monoterpenes & 1174 & $5.09 \pm 0.01$ & $5.00 \pm 0.76$ & $6.36 \pm 0.67$ & $5.25 \pm 0.25$ & $6.75 \pm 0.24$ & $3.24 \pm 0.27$ \\
\hline$\alpha$-terpineol & monocyclic monoterpenes & 1190 & $3.07 \pm 0.46$ & $3.46 \pm 0.47$ & $1.44 \pm 0.28$ & $3.57 \pm 0.12$ & $4.28 \pm 1.17$ & $3.54 \pm 0.11$ \\
\hline trans-piperitol & monocyclic monoterpenes & 1208 & $0.71 \pm 0.14$ & $0.44 \pm 0.03$ & $4.76 \pm 0.65$ & $0.19 \pm 0.00$ & $0.21 \pm 0.03$ & $0.19 \pm 0.15$ \\
\hline 6,7-epoxigeranial & acyclic monoterpenes & 1232 & $0.76 \pm 0.03$ & $0.65 \pm 0.01$ & $0.85 \pm 0.15$ & $0.39 \pm 0.11$ & $0.26 \pm 0.10$ & $0.32 \pm 0.01$ \\
\hline carvone & monocyclic monoterpenes & 1243 & $0.76 \pm 0.01$ & $0.3 \pm 0.01$ & $0.05 \pm 0.01$ & $0.66 \pm 0.06$ & $1.01 \pm 0.42$ & $0.45 \pm 0.02$ \\
\hline linalyl acetate & acyclic monoterpenes & 1247 & $2.49 \pm 0.20$ & $1.71 \pm 0.64$ & $2.74 \pm 0.70$ & $3.35 \pm 0.27$ & $2.82 \pm 0.69$ & $2.56 \pm 0.14$ \\
\hline carvacrol & monocyclic monoterpenes & 1296 & $9.18 \pm 2.01$ & $15.84 \pm 1.09$ & $6.52 \pm 2.26$ & $13.99 \pm 2.68$ & $5.49 \pm 0.75$ & $21.30 \pm 0.72$ \\
\hline$\beta$-bisabolene & sesquiterpenes & 1508 & $1.29 \pm 0.31$ & $0.67 \pm 0.01$ & $2.00 \pm 0.49$ & $1.50 \pm 0.23$ & $1.21 \pm 0.50$ & $1.70 \pm 0.08$ \\
\hline D-Hemp & Terpenes Class & RI & N2h & N6h & S2h & S6h & UAE & $\mathbf{M}$ \\
\hline$\alpha$-thuyene & bicyclic monoterpenes & 898 & $0.07 \pm 0.00$ & $0.06 \pm 0.02$ & $0.02 \pm 0.0$ & $0.02 \pm 0.0$ & - & $0.13 \pm 0.01$ \\
\hline$\alpha$-pinene & bicyclic monoterpenes & 915 & $0.09 \pm 0.00$ & $0.10 \pm 0.04$ & $0.40 \pm 0.1$ & $0.16 \pm 0.1$ & - & $0.22 \pm 0.04$ \\
\hline$\beta$-pinene & bicyclic monoterpenes & 959 & $0.16 \pm 0.01$ & $0.15 \pm 0.00$ & $0.34 \pm 0.0$ & $0.14 \pm 0.0$ & $0.28 \pm 0.03$ & $0.20 \pm 0.03$ \\
\hline$\beta$-myrcene & acyclic monoterpenes & 969 & $2.47 \pm 0.29$ & $0.78 \pm 0.11$ & $4.27 \pm 0.04$ & $3.03 \pm 0.8$ & - & $1.98 \pm 0.14$ \\
\hline D-limonene & monocyclic monoterpenes & 1011 & $0.65 \pm 0.34$ & $0.26 \pm 0.02$ & $0.55 \pm 0.01$ & $0.36 \pm 0.0$ & $0.10 \pm 0.03$ & $0.61 \pm 0.05$ \\
\hline eucaliptol & bicyclic monoterpenes & 1016 & $0.86 \pm 0.41$ & $0.46 \pm 0.07$ & $1.01 \pm 0.0$ & $0.74 \pm 0.1$ & $0.11 \pm 0.02$ & $2.14 \pm 0.26$ \\
\hline$\beta$-ocymene & acyclic monoterpenes & 1027 & $0.95 \pm 0.65$ & $0.15 \pm 0.04$ & $2.27 \pm 0.1$ & $1.11 \pm 0.3$ & $2.25 \pm 0.08$ & $0.52 \pm 0.01$ \\
\hline $\mathrm{X}$-terpinene & monocyclic monoterpenes & 1039 & $0.41 \pm 0.15$ & $0.89 \pm 0.11$ & $0.46 \pm 0.00$ & $0.25 \pm 0.00$ & $0.32 \pm 0.05$ & $1.66 \pm 0.07$ \\
\hline terpinolene & monocyclic monoterpenes & 1067 & $3.75 \pm 0.17$ & $0.37 \pm 0.04$ & $3.79 \pm 0.03$ & $5.05 \pm 0.9$ & $0.03 \pm 0.00$ & $2.15 \pm 0.72$ \\
\hline linalool & acyclic monoterpenes & 1079 & $4.93 \pm 0.30$ & $9.42 \pm 1.67$ & $2.69 \pm 0.04$ & $0.63 \pm 0.0$ & $0.26 \pm 0.04$ & $25.98 \pm 1.73$ \\
\hline
\end{tabular}


Table 2. Cont.

\begin{tabular}{|c|c|c|c|c|c|c|c|c|}
\hline L-trans-pinocarveol & bicyclic monoterpenes & 1109 & $0.87 \pm 0.36$ & $0.75 \pm 0.01$ & $0.42 \pm 0.1$ & $0.60 \pm 0.1$ & $0.81 \pm 0.15$ & $0.60 \pm 0.06$ \\
\hline cis-p-mentha-2,8-dien-1-ol & monocyclic monoterpenes & 1279 & $1.58 \pm 0.39$ & $1.23 \pm 0.10$ & $0.92 \pm 0.2$ & $1.35 \pm 0.0$ & $0.23 \pm 0.04$ & $1.20 \pm 0.01$ \\
\hline geranyl acetate & acyclic monoterpenes & 1372 & $1.00 \pm 0.16$ & $0.72 \pm 0.01$ & $1.46 \pm 0.59$ & $1.53 \pm 0.36$ & $0.96 \pm 0.63$ & $1.50 \pm 0.18$ \\
\hline caryophyllene & sesquiterpenes & 1390 & $51.85 \pm 2.57$ & $52.78 \pm 2.61$ & $52.44 \pm 0.1$ & $54.78 \pm 0.1$ & $40.00 \pm 0.36$ & $39.61 \pm 3.31$ \\
\hline$\alpha$-bergamotene & sesquiterpenes & 1400 & $5.86 \pm 1.26$ & $6.14 \pm 0.51$ & $4.83 \pm 0.0$ & $6.53 \pm 0.2$ & $4.79 \pm 0.22$ & $2.56 \pm 0.52$ \\
\hline cis- $\beta$-farnesene & sesquiterpenes & 1416 & $3.82 \pm 0.50$ & $3.54 \pm 0.42$ & $2.70 \pm 0.1$ & $4.20 \pm 0.3$ & $4.03 \pm 0.35$ & $2.18 \pm 0.18$ \\
\hline humulene & sesquiterpenes & 1423 & $13.60 \pm 1.81$ & $13.49 \pm 0.14$ & $12.11 \pm 0.2$ & $14.13 \pm 0.4$ & $12.25 \pm 0.99$ & $8.67 \pm 0.05$ \\
\hline aromadendrene & sesquiterpenes & 1428 & $1.85 \pm 0.18$ & $2.86 \pm 0.12$ & $2.35 \pm 0.0$ & $2.09 \pm 0.1$ & $3.16 \pm 0.19$ & $2.55 \pm 0.01$ \\
\hline$\beta$-selinene & sesquiterpenes & 1459 & $1.96 \pm 0.79$ & $2.81 \pm 0.17$ & $2.39 \pm 0.1$ & $0.96 \pm 0.6$ & $0.53 \pm 0.10$ & $3.37 \pm 0.39$ \\
\hline$\alpha$-selinene & sesquiterpenes & 1466 & $1.14 \pm 0.86$ & $1.91 \pm 0.15$ & $1.63 \pm 0.0$ & $1.12 \pm 0.1$ & $3.26 \pm 0.59$ & $2.30 \pm 0.25$ \\
\hline $\mathrm{V}$-cadinene & sesquiterpenes & 1408 & $0.32 \pm 0.11$ & $0.20 \pm 0.00$ & $0.40 \pm 0.0$ & $0.41 \pm 0.0$ & $0.37 \pm 0.07$ & $0.27 \pm 0.03$ \\
\hline guaia-3-9-diene & sesquiterpenes & 1413 & $1.08 \pm 0.11$ & $0.80 \pm 0.09$ & $1.60 \pm 0.0$ & $0.86 \pm 0.1$ & $0.00 \pm 0.00$ & $0.70 \pm 0.09$ \\
\hline selina-3,7(11)-diene & sesquiterpenes & 1418 & $0.91 \pm 0.08$ & $0.48 \pm 0.01$ & $1.65 \pm 0.1$ & $0.80 \pm 0.1$ & $1.19 \pm 0.21$ & $0.47 \pm 0.08$ \\
\hline caryophyllene oxide & sesquiterpenes & 1458 & $0.29 \pm 0.04$ & $0.27 \pm 0.01$ & $0.23 \pm 0.0$ & $0.29 \pm 0.1$ & $0.17 \pm 0.03$ & $0.20 \pm 0.02$ \\
\hline cis- $\alpha$-bisabolol & sesquiterpenes & 1589 & $0.05 \pm 0.01$ & $0.09 \pm 0.07$ & $0.04 \pm 0.0$ & $0.06 \pm 0.00$ & $0.32 \pm 0.06$ & $0.05 \pm 0.00$ \\
\hline ID-Coriander Seeds & Terpenes Class & RI & $\mathrm{N} 2 \mathrm{~h}$ & N6h & S2h & S6h & UAE & $\mathbf{M}$ \\
\hline$\alpha$-pinene & bicyclic monoterpenes & 915 & $0.15 \pm 0.04$ & $0.33 \pm 0.02$ & $0.49 \pm 0.07$ & $0.15 \pm 0.04$ & $0.50 \pm 0.01$ & $0.34 \pm 0.03$ \\
\hline 2-carene & bicyclic monoterpenes & 920 & $0.04 \pm 0.01$ & $0.07 \pm 0.02$ & $0.21 \pm 0.01$ & $0.04 \pm 0.01$ & $0.39 \pm 0.01$ & $0.04 \pm 0.01$ \\
\hline$p$-mentha-1,3,8-triene & monocyclic monoterpenes & 980 & $0.04 \pm 0.01$ & $0.05 \pm 0.01$ & $1.40 \pm 0.03$ & $0.04 \pm 0.01$ & - & $0.49 \pm 0.01$ \\
\hline$\beta$-terpinyl-acetate & monocyclic monoterpenes & 1343 & $0.04 \pm 0.01$ & $0.10 \pm 0.00$ & $0.04 \pm 0.01$ & $0.04 \pm 0.01$ & - & $0.13 \pm 0.02$ \\
\hline eucaliptol & bicyclic monoterpenes & 935 & - & - & $0.24 \pm 0.03$ & - & $0.33 \pm 0.00$ & $0.03 \pm 0.00$ \\
\hline$\beta$-myrcene & acyclic monoterpenes & 987 & $0.14 \pm 0.03$ & $0.21 \pm 0.00$ & $2.21 \pm 0.01$ & $0.15 \pm 0.03$ & $0.98 \pm 0.01$ & $0.23 \pm 0.02$ \\
\hline$\beta$-ocymene & acyclic monoterpenes & 1027 & $0.05 \pm 0.01$ & $0.09 \pm 0.01$ & $0.02 \pm 0.01$ & $0.05 \pm 0.01$ & - & $0.04 \pm 0.01$ \\
\hline $\mathrm{V}$-terpinene & monocyclic monoterpenes & 1053 & $0.15 \pm 0.02$ & $0.08 \pm 0.01$ & $2.24 \pm 0.03$ & $0.16 \pm 0.02$ & $1.41 \pm 0.00$ & $1.12 \pm 0.01$ \\
\hline trans-linalool oxide & monocyclic monoterpenes & 1083 & $0.74 \pm 0.03$ & $0.59 \pm 0.01$ & $7.50 \pm 0.04$ & $0.75 \pm 0.03$ & $0.55 \pm 0.04$ & $2.09 \pm 0.01$ \\
\hline terpinolene & monocyclic monoterpenes & 1085 & $0.04 \pm 0.01$ & $0.06 \pm 0.01$ & $0.03 \pm 0.01$ & $0.04 \pm 0.01$ & - & $0.03 \pm 0.01$ \\
\hline borneol & bicyclic monoterpenes & 1162 & $0.45 \pm 0.03$ & $0.39 \pm 0.02$ & $0.38 \pm 0.06$ & $0.45 \pm 0.03$ & - & $1.65 \pm 0.01$ \\
\hline linalool & acyclic monoterpenes & 1079 & $84.16 \pm 0.56$ & $72.55 \pm 0.03$ & $54.73 \pm 0.05$ & $83.99 \pm 0.56$ & $82.95 \pm 0.05$ & $78.20 \pm 0.02$ \\
\hline canfora & bicyclic monoterpenes & 1144 & $6.41 \pm 0.05$ & $3.26 \pm 0.02$ & $3.11 \pm 0.45$ & $6.47 \pm 0.05$ & $1.87 \pm 0.03$ & $7.90 \pm 0.02$ \\
\hline terpinen-4-ol & monocyclic monoterpenes & 1173 & $0.66 \pm 0.02$ & $0.78 \pm 0.00$ & $6.05 \pm 0.04$ & $0.67 \pm 0.02$ & $0.47 \pm 0.02$ & $0.76 \pm 0.01$ \\
\hline$\alpha$-terpineol & monocyclic monoterpenes & 1190 & $1.05 \pm 0.02$ & $2.14 \pm 0.03$ & $13.30 \pm 0.04$ & $1.06 \pm 0.02$ & $5.65 \pm 0.04$ & $1.19 \pm 0.01$ \\
\hline cis-geraniol & acyclic monoterpenes & 1248 & $3.96 \pm 0.14$ & $13.08 \pm 0.02$ & $0.25 \pm 0.01$ & $4.00 \pm 0.14$ & $0.67 \pm 0.04$ & $4.25 \pm 0.04$ \\
\hline lavandulyl acetate & acyclic monoterpenes & 1270 & $1.42 \pm 0.03$ & $5.50 \pm 0.01$ & $0.77 \pm 0.05$ & $1.44 \pm 0.03$ & $1.44 \pm 0.03$ & $1.28 \pm 0.02$ \\
\hline geranyl acetate & acyclic monoterpenes & 1372 & $0.48 \pm 0.03$ & $0.70 \pm 0.02$ & $2.77 \pm 0.01$ & $0.49 \pm 0.03$ & $0.58 \pm 0.03$ & $0.22 \pm 0.01$ \\
\hline
\end{tabular}

In the table: ID, component name; RI, retention index; N2h, RSLDE 2 h; N6h, RSLDE 6 h; S2h, Soxhlet 2 h; S6h, Soxhlet 6 h; UAE, ultrasound assisted extraction; M, maceration. 
Furthermore, 18 terpenoids were identified in coriander seeds extracts, all belonging to the monoterpenes class. The most abundant compound was linalool, which has antibacterial activity [33,34] and anti-tumorigenic potential [41]. Canfora and cis-geraniol were also present in smaller amounts; however, they have been reported to contribute to biological and antioxidant activity [37,42]. In literature, there are few studies of the chemical composition of extracts from coriander fruits; the terpenes profile found is similar to coriander seeds essential oils previously reported by Pellegrini et al. [43] and found in literature [44-46].

For each matrix, the SPME/GC-MS identified compounds were associated with four main classes of terpenes (class assignment of each compound is in Table 2). To explore potential correlations among the whole data set the PCA algorithm was used.

Figure 1 reports the PCA biplot obtained for the different terpenes classes (loadings), determined in extracts of thyme, hemp, and coriander (scores). The total variance explained was $70.51 \%$, with the first component accounting for $42.94 \%$ and the second for $27.57 \%$.

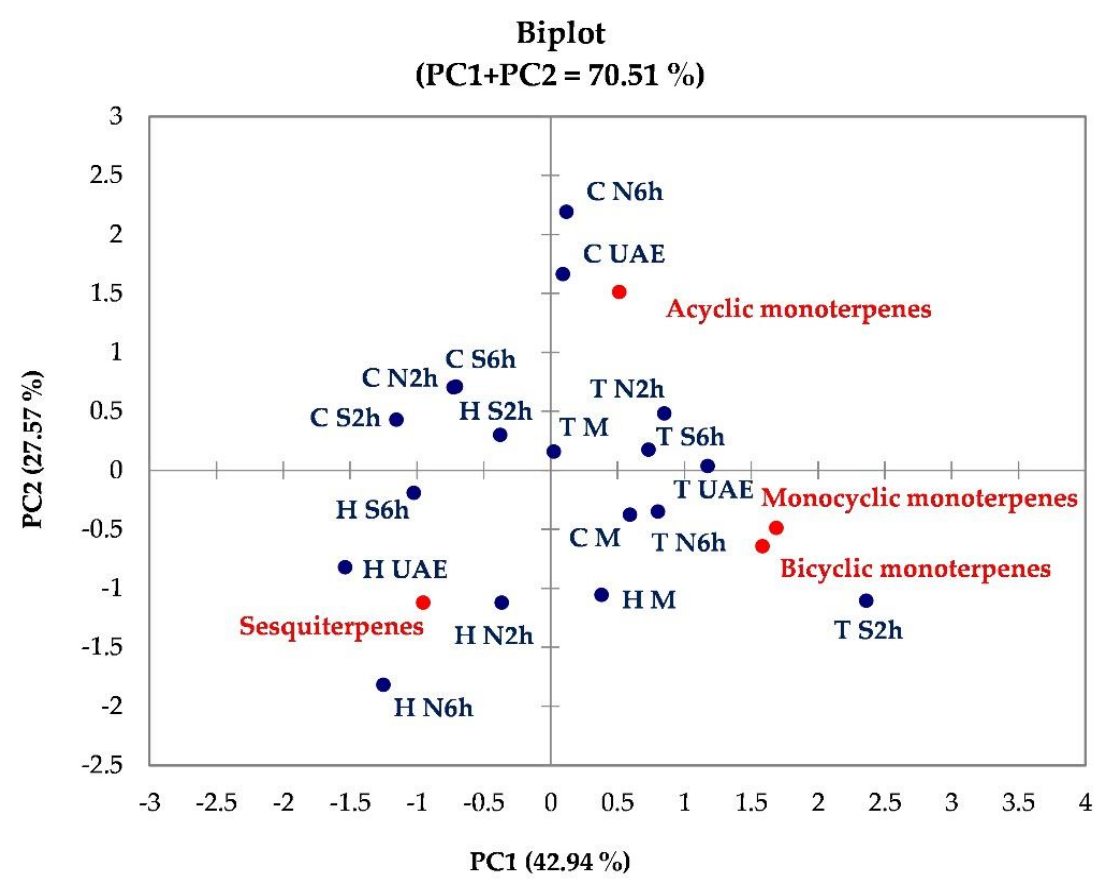

Figure 1. Biplot (scores and loadings) obtained from the PCA on data set of different extracts (rows) and terpenes classes analyzed (columns). In the Figure: T, thyme; H, hemp; C, coriander seeds; N2h, RSLDE 2 h; N6h, RSLDE 6 h; S2h, Soxhlet 2 h; S6h, Soxhlet 6 h; UAE, ultrasound assisted extraction; $\mathrm{M}$, maceration.

From the biplot, it is evident that thyme extract obtained from Soxhlet at $2 \mathrm{~h}$ of extraction (T S2h) is separated from all extracts based on the major content of monocyclic and bicyclic monoterpenes. These two classes of terpenes are strongly correlated. Sesquiterpenes were the most abundant compounds in hemp $6 \mathrm{~h}$ RSLDE extract (H N6h), while acyclic monoterpenes represented mainly the volatile fractions of coriander ultrasounds-assisted (C UAE) and $6 \mathrm{~h}$ RSLDE (C N6h) extracts. Based on the studied variables, from PCA is also evident the presence of different clusters; in particular, on PC1 the extracts C M, T N6h, T S6h, and T N2h are well grouped (positive correlation with PC1). The same applies for C S6h, C S2h, C N2h, H S2h, and H S6h (negative correlation with PC1).

To evaluate the influence of the extraction techniques on each matrix, the dataset was also processed through cluster analysis. Cluster analysis is a valid tool of multivariate analysis that has been already used and is useful to underline the differences among extraction techniques and conditions for the isolation of compounds from plant matrices [47,48]. Clusters were formed to contain four components (acyclic monoterpenes, monocyclic monoterpenes, bicyclic monoterpenes, sesquiterpenes). 
The dendrograms obtained from a cluster analysis of coriander, hemp, and thyme data are illustrated in Figures 2-4, respectively.

From the coriander dendrogram (Figure 2) is evident that the diagram is divided into three classes: one class comprising only M extract, and the other 2 constituted by N2h and Soxhlet at 2 and $6 \mathrm{~h}$ (S2h and S6h) extracts, and N6h and UAE extracts, respectively. A similar classification was achieved for hemp (Figure 3). In both dendrograms, the N6h/UAE class has large distance from the M class, meaning that these extraction techniques allowed for the isolation of different classes of terpenes. Indeed, N2h and Soxhlet at both 2 and $6 \mathrm{~h}$ of distillation time, have similar extraction patterns.

For thyme (Figure 4), a different distribution is obtained; one class consists of M, N2h, S6h, and UAE, the second and third consisting of only N6h and S2h, respectively. In this case, a larger distance of S2h from the first group was observed, because of the ability of this technique to extract more monocyclic and bicyclic monoterpenes, as already evidenced by PCA (Figure 1).

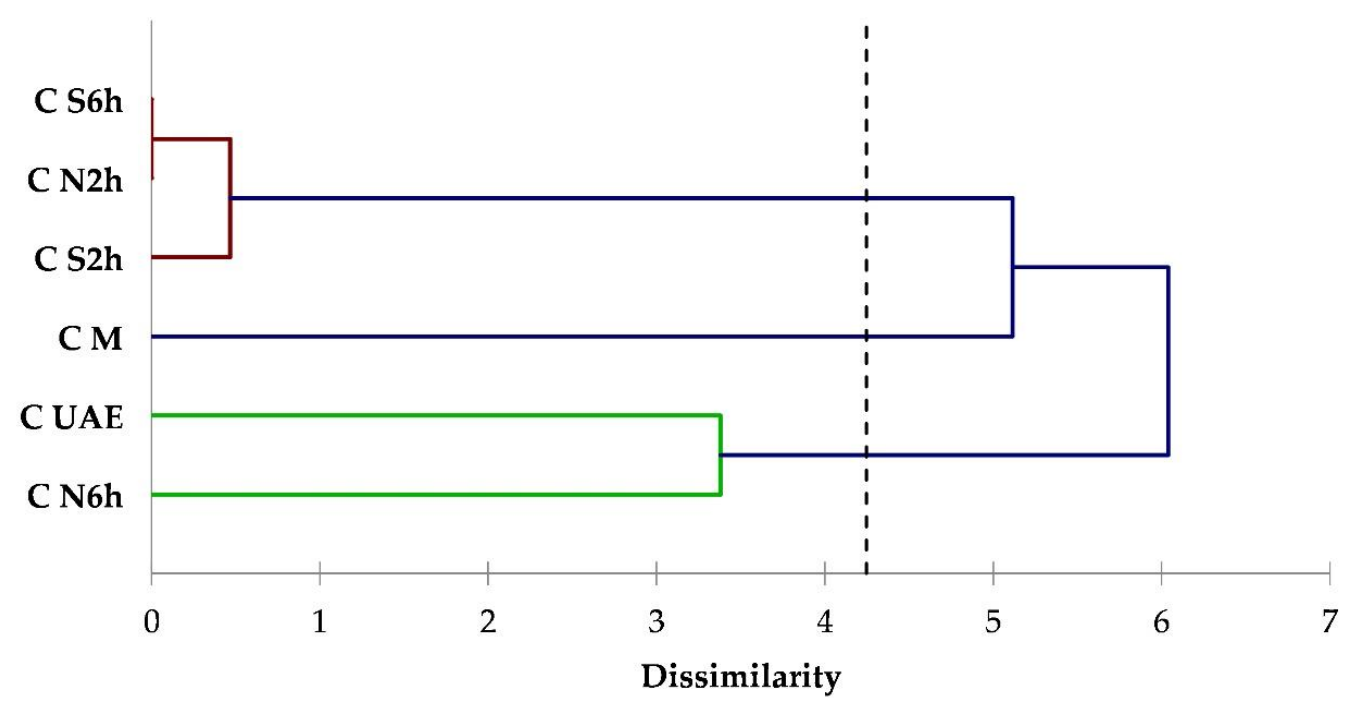

Figure 2. Dendrogram obtained from the cluster analysis based on terpenes classes data for coriander seeds extracts.

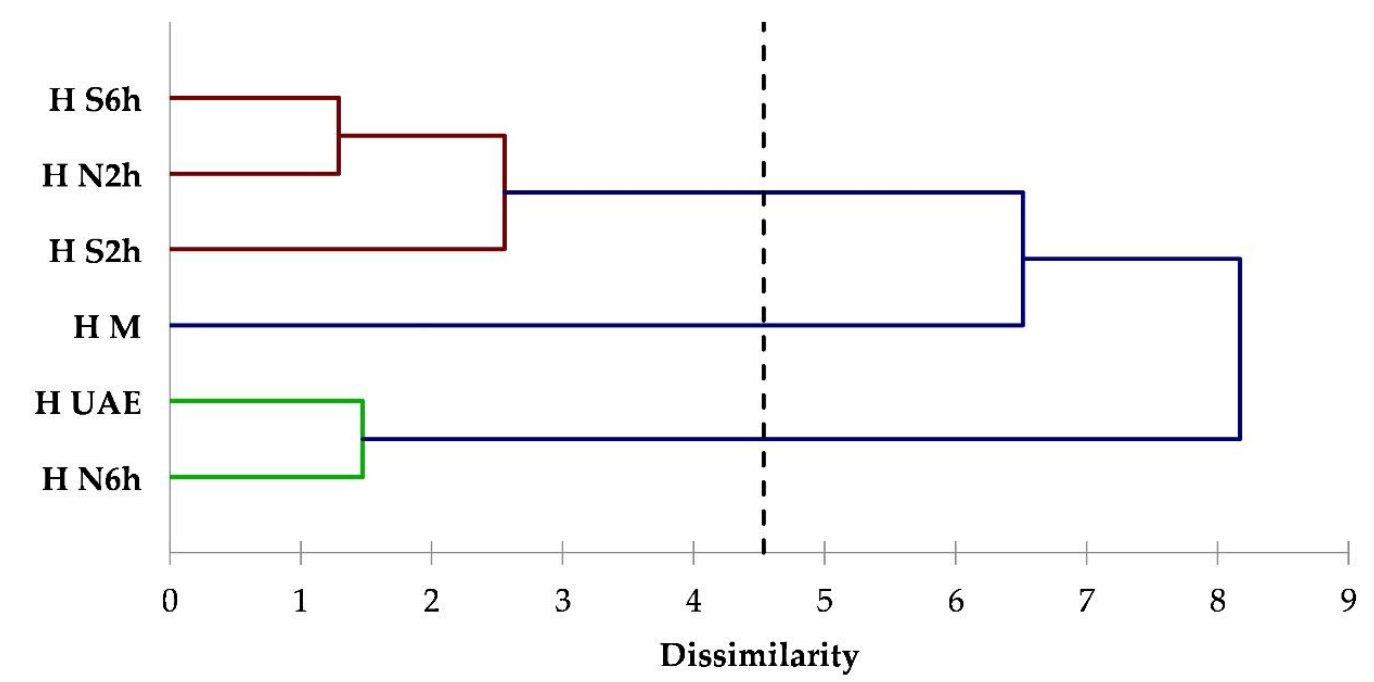

Figure 3. Dendrogram obtained from the cluster analysis based on terpenes classes data for hemp extracts. 


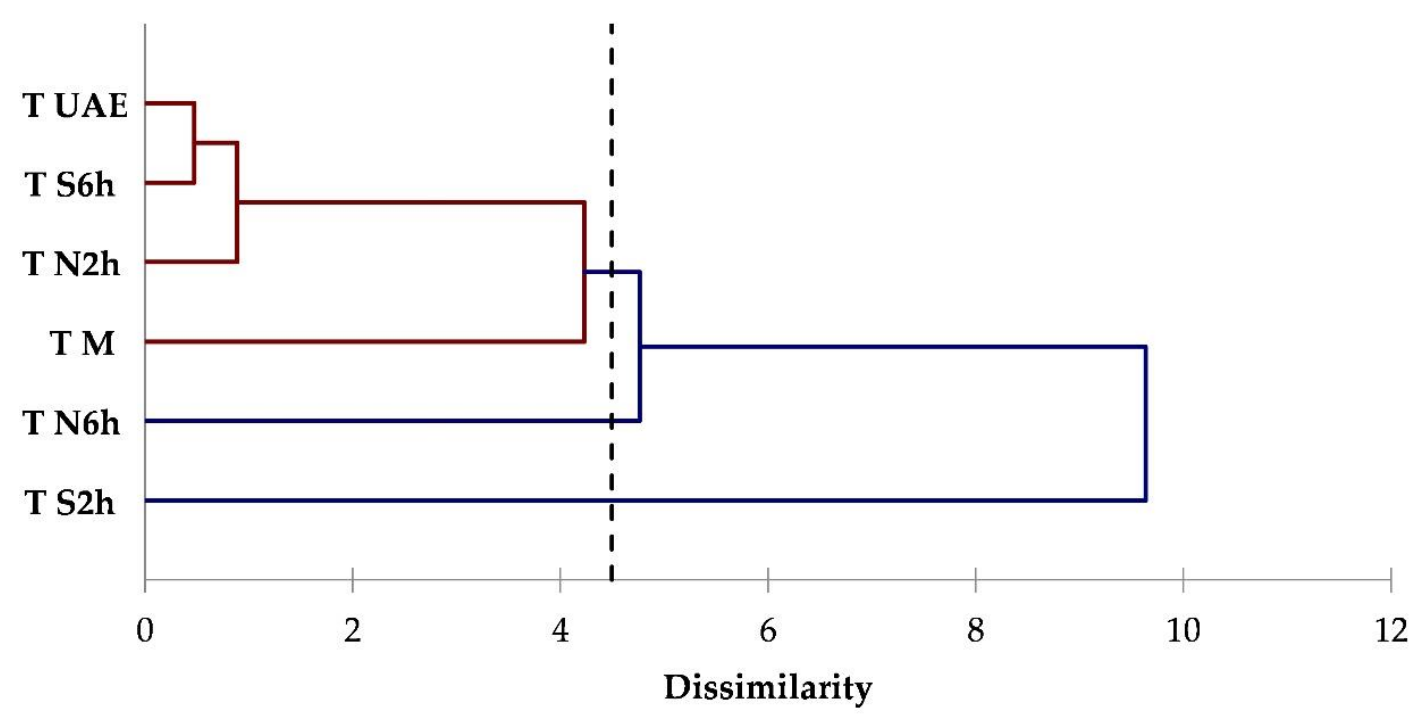

Figure 4. Dendrogram obtained from the cluster analysis based on terpenes classes data for thyme extracts.

The cluster analyses of terpenes allowed to underline that there were not clear patterns of extraction that can help in selecting a particular technique for the extraction of a certain class of terpenes. This is mainly related to the variables that occur before, during and after the extraction process and that influence the outcomes [8]. Anyhow, for all matrices the RSLDE at $2 \mathrm{~h}$ of extraction is always clustered with Soxhlet at 6 hours, indicating that, regardless of matrix nature, the extraction patterns are very similar for the two approaches.

\subsection{Polyphenolic Composition}

The HPLC-UV qualitative and quantitative analysis results of the extracts are presented in Table 3.

Eight phenolic acids, (i.e., gallic acid, $p$-OH-benzoic acid, chlorogenic acid, vanillic acid, caffeic acid, syringic acid, ferulic acid, and rosmarinic acid), one phenolic monoterpene (carvacrol) and two flavonoids (i.e., luteolin and apigenin) were identified in thyme. Rosmarinic acid was the compound with the highest concentration in all extracts. Rosmarinic acid is known as one of the main constituents of thyme and it has been recognized for antioxidant, antiviral, anti-inflammatory, antibacterial, and immunostimulant activities [49,50]. UAE and M extracts were significantly poorer of polyphenols, except for the highest UAE luteolin content; however, they were the only extracts containing some phenolic acids (i.e., vanillic and caffeic acids in UAE, syringic acid in M). RSLDE exhibited improved extraction of $p$-OH-benzoic and chlorogenic acids. A non-univocal effect of the increase of the extraction time was observed, as some components have increased and others decreased. In line with our findings, the decrease in content of rosmarinic acid and luteolin at prolonged extraction time has been reported by other authors [51,52]. Besides the extraction operative conditions like solvent, temperature, and time, the stability of natural products in certain conditions is a variable that may influence the chemical composition of the extract. The bioactive compound, during the extraction procedure, are exposed to chemical reactions with solvent and/or other components in the solution that rearrange chemical structures. Chemical alterations occur also after the extraction process, due to manipulation (e.g., solvent removal) and/or conservation conditions (e.g., compounds breakdown by oxidation or light) [53,54]. 
Table 3. Contents of phenolic compounds ( $\mu \mathrm{g} / \mathrm{g}$ dry extract).

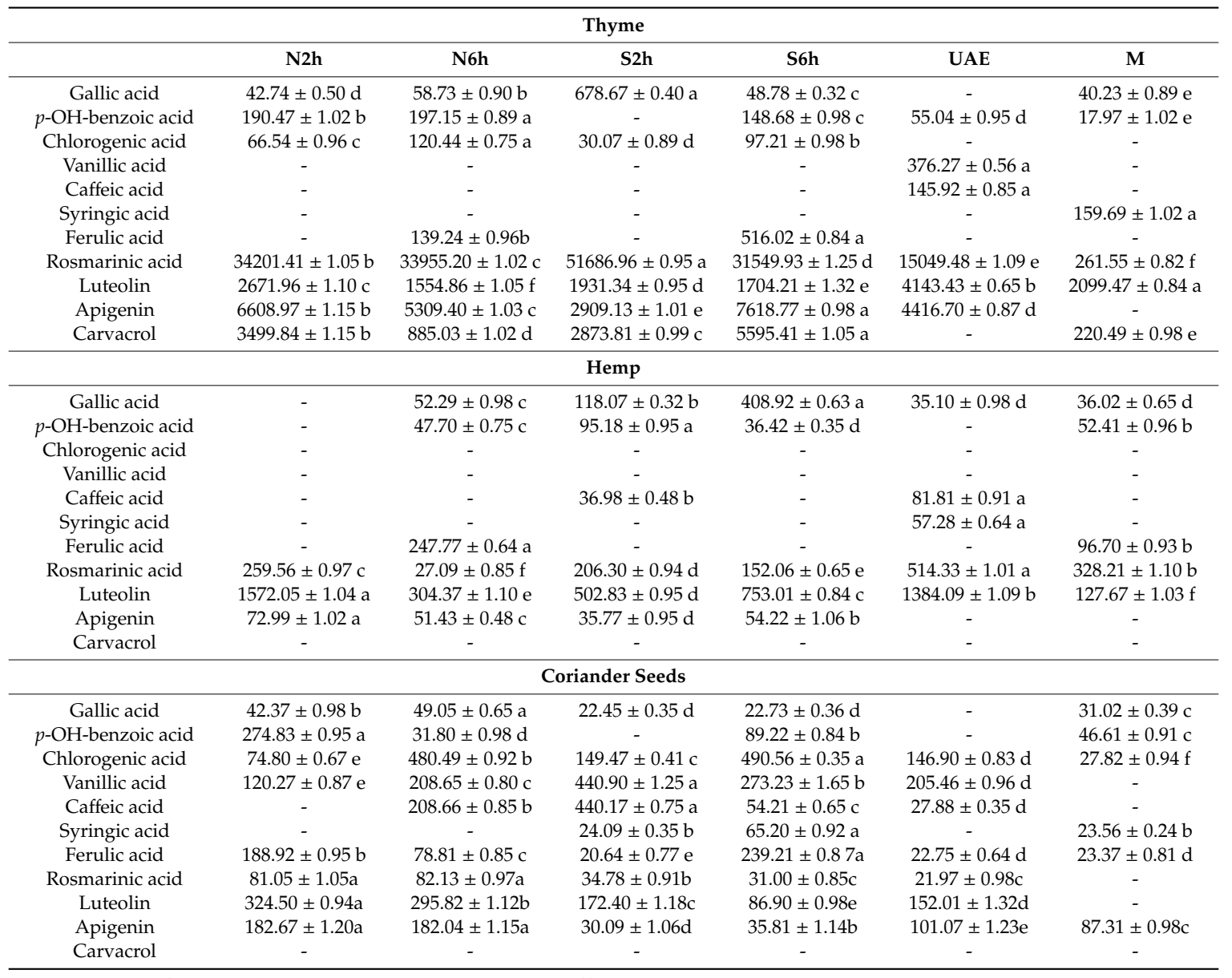

Results followed by the same case-letter are not different according to Tukey's HSD post-hoc test $(p>0.05)$.

Three phenolic acids (i.e., gallic acid, $p$-OH-benzoic acid, and rosmarinic acid) and two flavonoids (i.e., luteolin and apigenin) were mainly found in hemp. The most abundant component was luteolin, a flavonoid with antioxidant [55], anti-inflammatory and antiallergic [56] activity. Among the few literature studies on the identification of polyphenols in hemp extracts, the presence in hemp essential oil of gallic acid and $p$-OH benzoic acid has been already reported [57]. Considering the different extraction techniques, N2h was the extract richest in the three main compounds: rosmarinic acid, luteolin, and apigenin. N6h indicated that the increase in extraction time allowed the recovery of gallic acid, $p-\mathrm{OH}$-benzoic acid, and ferulic acid, with a loss in the main components. Indeed, the increase in Soxhlet extraction time from $2 \mathrm{~h}$ to $6 \mathrm{~h}$ led to an increase in the concentration of gallic acid and of the two flavonoids luteolin and apigenin, accompanied by the decrease of $p$-OH-benzoic acid and rosmarinic acid. $\mathrm{M}$ was the poorest extract compared to other techniques.

Similarly to thyme, eight phenolic acids (i.e., gallic acid, $p$-OH-benzoic acid, chlorogenic acid, vanillic acid, caffeic acid, syringic acid, ferulic acid, and rosmarinic acid) and two flavonoids (i.e., luteolin and apigenin) were detected in coriander seeds. Barros and collaborators [58] reported a similar profile in the analysis of polyphenols of coriander seeds. However, gallic acid, luteolin, and apigenin were not detected. Msaada and collaborators [59] described a similar composition, with a higher number of flavonoids components (quercetin, rutin, luteolin, apigenin, and kaempferol). Among the different extraction techniques, RSLDE extracts were richer in gallic, $p$-OH-benzoic, and rosmarinic phenolic acids and in luteolin and apigenin, with a positive effect of longer extraction time on their concentration, except for $p$-OH-benzoic acid and luteolin. On the contrary, Soxhlet extracts showed 
higher concentrations of chlorogenic, vanillic, and caffeic acids, in addition to the presence of syringic acid, that was not detected in N2h and N6h.

\subsection{Total Phenolic Content and Antioxidant Activity}

Total phenolic content (TPC) and antioxidant activity (AOC-FRAP, DPPH;, $\mathrm{ABTS}^{+}$) data are reported in Table 4. The different extraction techniques were compared using an analysis of variance (ANOVA).

Table 4. Total phenolic content (TPC) and antioxidant activity. Ferric Reducing Antioxidant Power (FRAP), and Trolox Equivalent Antioxidant Capacity with 2,2-diphenyl-1-picrylhydrazyl (DPPH), and 2,2'-azinobis-(3-ethylbenzothiazoline-6-sulfonic acid $\left(\mathrm{ABTS}^{+}\right.$) of extracts of different techniques.

\begin{tabular}{|c|c|c|c|c|}
\hline & $\begin{array}{c}\text { TPC } \\
\text { (mg GAE/g Dry Extract) }\end{array}$ & $\begin{array}{c}\text { FRAP } \\
\text { (mg TE/g Dry Extract) }\end{array}$ & $\begin{array}{c}\text { DPPH } \\
\text { (mg TE/g Dry Extract) }\end{array}$ & $\begin{array}{c}\text { ABTS }^{+} \\
\text {(mg TE/g Dry Extract) }\end{array}$ \\
\hline \multicolumn{5}{|c|}{ Thyme } \\
\hline $\mathrm{N} 2 \mathrm{~h}$ & $141.86 \pm 7.85 b$ & $63.86 \pm 0.29 b$ & $108.17 \pm 0.08 \mathrm{a}$ & $23.62 \pm 8.20 \mathrm{~d}$ \\
\hline N6h & $179.67 \pm 12.71 \mathrm{a}$ & $66.95 \pm 5.74 b$ & $108.52 \pm 0.16 \mathrm{a}$ & $52.12 \pm 0.11 b c$ \\
\hline $\mathrm{S} 2 \mathrm{~h}$ & $157.86 \pm 1.71 \mathrm{~b}$ & $68.48 \pm 1.23 \mathrm{ab}$ & $108.57 \pm 0.08 \mathrm{a}$ & $49.07 \pm 0.11 \mathrm{c}$ \\
\hline S6h & $154.26 \pm 11.23 \mathrm{~b}$ & $73.53 \pm 1.29 \mathrm{a}$ & $107.19 \pm 1.22 \mathrm{a}$ & $134.46 \pm 0.22 \mathrm{a}$ \\
\hline UAE & $107.91 \pm 9.01 \mathrm{c}$ & $40.60 \pm 1.04 c$ & $98.50 \pm 1.71 b$ & $53.72 \pm 0.34 b c$ \\
\hline $\mathrm{M}$ & $142.08 \pm 1.69 \mathrm{~b}$ & $40.25 \pm 2.93 c$ & $92.60 \pm 4.33 c$ & $57.56 \pm 0.34 \mathrm{~b}$ \\
\hline \multicolumn{5}{|c|}{ Hemp } \\
\hline $\mathrm{N} 2 \mathrm{~h}$ & $140.25 \pm 2.56 \mathrm{a}$ & $81.073 \pm 2.31 \mathrm{ab}$ & $34.02 \pm 1.86 \mathrm{~b}$ & $557.16 \pm 6.57 a$ \\
\hline N6h & $139.52 \pm 2.49 \mathrm{a}$ & $80.21 \pm 1.76 \mathrm{abc}$ & $33.76 \pm 0.89 b$ & $485.10 \pm 4.16 \mathrm{~b}$ \\
\hline $\mathrm{S} 2 \mathrm{~h}$ & $120.55 \pm 5.42 b$ & $74.66 \pm 0.81 \mathrm{~d}$ & $29.92 \pm 0.32 \mathrm{~d}$ & $394.39 \pm 3.41 c$ \\
\hline S6h & $124.25 \pm 3.61 b$ & $78.69 \pm 1.88 \mathrm{bc}$ & $31.54 \pm 0.08 \mathrm{~cd}$ & $433.22 \pm 19.81 b c$ \\
\hline UAE & $110.30 \pm 3.71 c$ & $77.22 \pm 0.92 \mathrm{~cd}$ & $45.04 \pm 1.23 \mathrm{a}$ & $381.26 \pm 9.05 \mathrm{~cd}$ \\
\hline $\mathrm{M}$ & $125.12 \pm 3.54 b$ & $83.14 \pm 1.63 \mathrm{a}$ & $32.43 \pm 0.32 b c$ & $502.16 \pm 5.62 \mathrm{ab}$ \\
\hline \multicolumn{5}{|c|}{ Coriander Seeds } \\
\hline $\mathrm{N} 2 \mathrm{~h}$ & $19.87 \pm 1.52 b$ & $18.43 \pm 0.15 a$ & $147.60 \pm 3.97 b$ & $48.05 \pm 1.60 \mathrm{a}$ \\
\hline N6h & $17.67 \pm 0.47 c$ & $12.69 \pm 0.39 \mathrm{~d}$ & $163.55 \pm 2.94 b$ & $46.48 \pm 1.78 \mathrm{a}$ \\
\hline $\mathrm{S} 2 \mathrm{~h}$ & $15.54 \pm 0.57 \mathrm{~d}$ & $15.22 \pm 0.39 \mathrm{bc}$ & $128.84 \pm 3.94 b$ & $21.90 \pm 2.00 \mathrm{~b}$ \\
\hline S6h & $24.36 \pm 1.01 \mathrm{a}$ & $16.91 \pm 2.34 \mathrm{ab}$ & $150.61 \pm 4.92 b$ & $23.85 \pm 1.60 b$ \\
\hline UAE & $3.01 \pm 0.61 \mathrm{e}$ & $13.45 \pm 1.26 \mathrm{~cd}$ & $219.95 \pm 2.44 \mathrm{a}$ & $1.12 \pm 0.14 \mathrm{~d}$ \\
\hline $\mathrm{M}$ & $18.95 \pm 0.11 b c$ & $14.40 \pm 0.09 \mathrm{~cd}$ & $177.23 \pm 1.46 \mathrm{ab}$ & $5.64 \pm 1.04 \mathrm{c}$ \\
\hline
\end{tabular}

Results followed by the same case-letter are not different according to Tukey's HSD post-hoc test $(p>0.05)$.

The comparison with literature indicates that our data are in accordance with those reported by different authors [60-64].

The influence of the extraction time on TPC was more evident for thyme RSLDE extracts and coriander Soxhlet extracts, with improved extraction for longer times ( $6 \mathrm{~h}$ with respect to $2 \mathrm{~h}$ ). In the comparison of the different techniques, the highest values were registered for RSLDE extracts for thyme and hemp, at $6 \mathrm{~h}$ and $2 \mathrm{~h}$, respectively. Indeed, in the case of coriander seeds, Soxhlet extraction at $6 \mathrm{~h}$ was more efficient. For all plant matrices, UAE extracts had the lowest TPC values.

Concerning AOC (Table 4) of hemp and coriander seeds, a higher activity for the $2 \mathrm{~h}$ RSLDE extracts in all spectrophotometric assays was achieved, except for the DPPH: The highest antiradical activity was obtained for UAE extracts. Moreover, in the case of hemp, M demonstrated good activity, similarly to RSLDE extracts.

It is known that the differences in the antioxidant activity might be related to the different availability of extractable components, resulting from the varied chemical composition of plants [65]. The amount of the antioxidant components that can be extracted from a plant material is mainly affected by the strength of the extraction procedure and may vary from sample to sample. Usually, the TPC and AOC of extracts obtained by reflux extraction technique (e.g., Soxhlet) are lower than 
other methods [27], in contrast to the trends noted for extraction yields. This decrease can be attributed to the thermal decomposition of some antioxidants at the temperatures used in the process. Several studies reported that thermal processing conditions might result in the loss of natural antioxidants because heat may accelerate oxidation and other degradation reactions $[27,66,67]$.

In this work, a different behavior was observed; for thyme, in fact, Soxhlet at $6 \mathrm{~h}$ was the best extract in terms of antioxidant activity. This indicates that for this aromatic plant, hot solvent systems under reflux state are more efficient for the recovery of antioxidant components. Dutra et al. [68] reported that among different extraction techniques (i.e., reflux, maceration, ultrasound, heating plate), extraction made under reflux using ethanol/water $(70: 30, v / v)$ offered the highest polyphenol levels in Pterodon emarginatus vogel seeds. This was attributed to the effective extraction under reflux conditions, leading to a higher release of some bound phenolics and with an increase of antioxidant activity [69].

To highlight the comparison of the different extraction techniques, for each plant matrix, the best performing extracts in TPC and antioxidant activity assays are collected in Table 5.

Table 5. Extracts with the highest value of total phenolic content and antioxidant activity

\begin{tabular}{ccccc}
\hline & TPC & FRAP & DPPH & ABTS $^{+}$ \\
\hline Thyme & N6h & S6h & N2h/N6h/S2h/S6h & S6h \\
Hemp & N2h/N6h & M & UAE & N2h \\
Coriander seeds & S6h & N2h & UAE & N2h/N6h \\
\hline
\end{tabular}

Table 5 clearly shows that all the extraction techniques are very efficient methods for the recovery of bioactive compounds from plant matrices, particularly to produce extracts retaining good antioxidant capacity. It is worth to notice, however, that more than half of the cases are carried out using unconventional techniques, (N2h/N6h and UAE), thus reducing solvent and energy consumption.

\section{Conclusions}

In this study, extracts have been obtained from three plant species T. vulgaris, C. sativa, and C. sativum cultivated in the Abruzzo region. Four different extraction methods were applied to recover the plants' bioactive components, two conventional, namely the maceration and Soxhlet technique, and two unconventional, namely the ultrasound assisted extraction and the rapid solid-liquid dynamic extraction, performed by Naviglio Extractor ${ }^{\circledR}$. Moreover, for the Soxhlet and RSLDE techniques, the effect of the extraction time was also investigated.

All the obtained extracts are rich in bioactive compounds and display good antioxidant properties. Although the results do not show univocal trends, slightly higher performances are observed for the extracts obtained by the unconventional RSLDE and UAE techniques.

Given the limited effect of the increase in extraction time, the RSLDE technique performed by Naviglio Extractor ${ }^{\circledR}$ at $2 \mathrm{~h}$ of extraction time can be considered a good standardized method to obtain extracts with interesting in vitro antioxidant activity and potential candidates as natural preservatives in food.

Author Contributions: Conceptualization A.R. and C.L.S.; methodology and investigation, S.P. and M.P.; data curation, S.P., A.R., and M.P.; writing—original draft preparation, S.P., and M.P.; writing—review and editing, A.R. and D.C.; supervision, A.R., C.L.S., and D.C. All authors have read and agreed to the published version of the manuscript.

Funding: This research has been supported by the grant of Regione Abruzzo, for the project "PSR 2017-2013 Misura 1.2.4" cod. CUA 2446850691, title "Sviluppo di sistemi convenzionali e innovazioni per la produzione di composti bioattivi da materie prime vegetali per l'impiego nel settore alimentare", and by the FARDIB Research Project of the University of Teramo.

Conflicts of Interest: The authors declare no conflict of interest. 


\section{References}

1. Sasidharan, S.; Chen, Y.; Saravanan, D.; Sundram, K.; Latha, L.Y. Extraction, isolation and characterization of bioactive compounds from plants' extracts. Afr. J. Tradit. Complement Altern. Med. 2011, 8, 1-10. [CrossRef] [PubMed]

2. Tajkarimi, M.; Ibrahim, S.A.; Cliver, D. Antimicrobial herb and spice compounds in food. Food Control 2010, 21, 1199-1218. [CrossRef]

3. Gonzalez-Burgos, E.; Gomez-Serranillos, M. Terpene compounds in nature: A review of their potential antioxidant activity. Curr. Med. Chem. 2012, 19, 5319-5341. [CrossRef] [PubMed]

4. Briskin, D.P. Medicinal plants and phytomedicines. Linking plant biochemistry and physiology to human health. Plant Physiol. 2000, 124, 507-514. [CrossRef] [PubMed]

5. Tomaino, A.; Cimino, F.; Zimbalatti, V.; Venuti, V.; Sulfaro, V.; De Pasquale, A.; Saija, A. Influence of heating on antioxidant activity and the chemical composition of some spice essential oils. Food Chem. 2005, 89, 549-554. [CrossRef]

6. Altemimi, A.; Lakhssassi, N.; Baharlouei, A.; Watson, D.G.; Lightfoot, D.A. Phytochemicals: Extraction, isolation, and identification of bioactive compounds from plant extracts. Plants 2017, 6, 42. [CrossRef]

7. Smith, R.M. Before the injection-Modern methods of sample preparation for separation techniques. J. Chromatogr. A 2003, 1000, 3-27. [CrossRef]

8. Azmir, J.; Zaidul, I.; Rahman, M.; Sharif, K.; Mohamed, A.; Sahena, F.; Jahurul, M.; Ghafoor, K.; Norulaini, N.; Omar, A. Techniques for extraction of bioactive compounds from plant materials: A review. J. Food Eng. 2013, 117, 426-436. [CrossRef]

9. De Castro, M.L.; Priego-Capote, F. Soxhlet extraction: Past and present Panacea. J. Chromatogr. A 2010, 1217, 2383-2389. [CrossRef]

10. Belwal, T.; Ezzat, S.M.; Rastrelli, L.; Bhatt, I.D.; Daglia, M.; Baldi, A.; Prasad Devkota, H.; Erdogan Orhan, I.; Kumar Patra, J.; Das, G.; et al. A critical analysis of extraction techniques used for botanicals: Trends, priorities, industrial uses and optimization strategies. Trends Anal. Chem. 2018, 100, 82-102. [CrossRef]

11. Chemat, F.; Rombaut, N.; Sicaire, A.-G.; Meullemiestre, A.; Fabiano-Tixier, A.-S.; Abert-Vian, M. Ultrasound assisted extraction of food and natural products. Mechanisms, techniques, combinations, protocols and applications A Review. Ultrason. Sonochem. 2017, 34, 540-560. [CrossRef] [PubMed]

12. Naviglio, D.; Scarano, P.; Ciaravolo, M.; Gallo, M. Rapid Solid-Liquid Dynamic Extraction (RSLDE): A powerful and greener alternative to the latest solid-liquid extraction techniques. Foods 2019, 8, 245. [CrossRef] [PubMed]

13. Skenderidis, P.; Petrotos, K.; Giavasis, I.; Hadjichristodoulou, C.; Tsakalof, A. Optimization of ultrasound assisted extraction of of goji berry (Lycium barbarum) fruits and evaluation of extracts' bioactivity. J. Food Process Eng. 2017, 40, e12522. [CrossRef]

14. Medina-Torres, N.; Ayora-Talavera, T.; Espinosa-Andrews, H.; Sánchez-Contreras, A.; Pacheco, N. Ultrasound Assisted Extraction for the Recovery of Phenolic Compounds from Vegetable Sources. Agronomy 2017, 7, 47. [CrossRef]

15. Naviglio, D. Naviglio's principle and presentation of an innovative solid-liquid extraction technology: Extractor Naviglioß. Anal. Lett. 2003, 36, 1647-1659. [CrossRef]

16. Gallo, M.; Vitulano, M.; Andolfi, A.; Della Greca, M.; Conte, E.; Ciaravolo, M.; Naviglio, D. Rapid Solid-Liquid Dynamic Extraction (RSLDE): A new rapid and greener method for extracting two steviol glycosides (Stevioside and Rebaudioside A) from stevia leaves. Plant Foods Hum. Nutr. 2017, 72, 141-148. [CrossRef]

17. Ferrara, L.; Naviglio, D.; Gallo, M. Extraction of bioactive compounds of saffron (Crocus sativus L.) by ultrasound assisted extraction (UAE) and by rapid solid-liquid dynamic extraction (RSLDE). Eur. Sci. J. 2014, 10, 1-13.

18. Cozzolino, I.; Vitulano, M.; Conte, E.; D’Onofrio, F.; Aletta, L.; Ferrara, L.; Andolfi, A.; Naviglio, D.; Gallo, M. Extraction and curcuminoids activity from the roots of Curcuma longa by RSLDE using the Naviglio extractor. Eur. Sci. J. (Special Edition) 2016, 12, 119-127.

19. Gallo, M.; Formato, A.; Ciaravolo, M.; Langella, C.; Cataldo, R.; Naviglio, D. A water extraction process for lycopene from tomato waste using a pressurized method: An application of a numerical simulation. Eur. Food Res. Technol. 2019, 245, 1767-1775. [CrossRef] 
20. Lee, K.-G.; Shibamoto, T. Antioxidant properties of aroma compounds isolated from soybeans and mung beans. J. Agric. Food Chem. 2000, 48, 4290-4293. [CrossRef]

21. Singleton, V.L.; Rossi, J.A. Colorimetry of total phenolics with phosphomolybdic-phosphotungstic acid reagents. Am. J. Enol. Vitic. 1965, 16, 144-158.

22. Brand-Williams, W.; Cuvelier, M.-E.; Berset, C. Use of a free radical method to evaluate antioxidant activity. LWT-Food Sci. Technol. 1995, 28, 25-30. [CrossRef]

23. Gullon, B.; Pintado, M.E.; Barber, X.; Fernández-López, J.; Pérez-Álvarez, J.A.; Viuda-Martos, M. Bioaccessibility, changes in the antioxidant potential and colonic fermentation of date pits and apple bagasse flours obtained from co-products during simulated in vitro gastrointestinal digestion. Food Res. Int. 2015, 78, 169-176. [CrossRef] [PubMed]

24. Oyaizu, M. Studies on products of browning reaction. Jpn. J. Nutr. Diet. 1986, 44, 307-315. [CrossRef]

25. Zekovic, Z.P. Analysis of thyme (Thymus vulgaris L.) extracts. Acta Period. Technol. Yugosl. 2000, 31, 617-622.

26. Zhang, Z.-M.; Li, G.-K. A preliminary study of plant aroma profile characteristics by a combination sampling method coupled with GC-MS. Microchem. J. 2007, 86, 29-36. [CrossRef]

27. Sultana, B.; Anwar, F.; Ashraf, M. Effect of extraction solvent/technique on the antioxidant activity of selected medicinal plant extracts. Molecules 2009, 14, 2167-2180. [CrossRef] [PubMed]

28. Wei, J.-N.; Liu, Z.-H.; Zhao, Y.-P.; Zhao, L.-L.; Xue, T.-K.; Lan, Q.-K. Phytochemical and Bioactive Profile of Coriandrum sativum L. Food Chem. 2019, 286, 260-267. [CrossRef]

29. Devi, V.; Khanam, S. Comparative study of different extraction processes for hemp (Cannabis sativa) seed oil considering physical, chemical and industrial-scale economic aspects. J. Clean. Prod. 2019, 207, 645-657. [CrossRef]

30. Guillen, M.; Manzanos, M. Study of the composition of the different parts of a Spanish Thymus vulgaris L. plant. Food Chem. 1998, 63, 373-383. [CrossRef]

31. Cappelletto, P.; Brizzi, M.; Mongardini, F.; Barberi, B.; Sannibale, M.; Nenci, G.; Poli, M.; Corsi, G.; Grassi, G.; Pasini, P. Italy-grown hemp: Yield, composition and cannabinoid content. Ind. Crops Prod. 2001, 13, 101-113. [CrossRef]

32. Butt, A.S.; Nisar, N.; Ghani, N.; Altaf, I.; Mughal, T.A. Isolation of thymoquinone from Nigella sativa L. and Thymus vulgaris L., and its anti-proliferative effect on HeLa cancer cell lines. Trop. J. Pharm. Res. 2019, 18, 37-42. [CrossRef]

33. Karam, L.; Roustom, R.; Abiad, M.G.; El-Obeid, T.; Savvaidis, I.N. Combined effects of thymol, carvacrol and packaging on the shelf-life of marinated chicken. Int. J. Food Microbiol. 2019, 291, 42-47. [CrossRef] [PubMed]

34. Flores, P.I.G.; Valenzuela, R.B.; Ruiz, L.D.; López, C.M.; Cháirez, F.E. Antibacterial activity of five terpenoid compounds: Carvacrol, limonene, linalool, $\alpha$-terpinene and thymol. Trop. Subtrop. Agroecosyst. 2019, 22, 443-450.

35. Agatonovic-Kustrin, S.; Kustrin, E.; Morton, D.W. Essential oils and functional herbs for healthy aging. Neural Regen. Res. 2019, 14, 441-445. [CrossRef] [PubMed]

36. Andre, C.M.; Hausman, J.-F.; Guerriero, G. Cannabis sativa: The plant of the thousand and one molecules. Front. Plant Sci. 2016, 7, 1-17. [CrossRef]

37. Nuutinen, T. Medicinal properties of terpenes found in Cannabis sativa and Humulus lupulus. Eur. J. Med. Chem. 2018, 157, 198-228. [CrossRef]

38. Tambe, Y.; Tsujiuchi, H.; Honda, G.; Ikeshiro, Y.; Tanaka, S. Gastric Cytoprotection of the Non-Steroidal Anti-Inflammatory Sesquiterpene, $\beta$-Caryophyllene. Planta Med. 1996, 62, 469-470. [CrossRef]

39. Pellati, F.; Brighenti, V.; Sperlea, J.; Marchetti, L.; Bertelli, D.; Benvenuti, S. New methods for the comprehensive analysis of bioactive compounds in Cannabis sativa L.(hemp). Molecules 2018, 23, 2639. [CrossRef]

40. Stenerson, K.K.; Halpenny, M.R. Analysis of Terpenes in Cannabis Using Headspace Solid-Phase Microextraction and GC-MS. LCGC North Am. 2017, 35, 28.

41. Jana, S.; Patra, K.; Sarkar, S.; Jana, J.; Mukherjee, G.; Bhattacharjee, S.; Mandal, D.P. Antitumorigenic potential of linalool is accompanied by modulation of oxidative stress: An in vivo study in sarcoma-180 solid tumor model. Nutr. Cancer 2014, 66, 835-848. [CrossRef] [PubMed]

42. Chen, W.; Viljoen, A. Geraniol-A review of a commercially important fragrance material. South Afr. J. Bot. 2010, 76, 643-651. [CrossRef] 
43. Pellegrini, M.; Rossi, C.; Palmieri, S.; Maggio, F.; Chaves, C.; Sterzo, C.L.; Paparella, A.; de Medici, D.; Ricci, A.; Serio, A. Salmonella enterica Control in Stick Carrots through Incorporation of Coriander Seeds Essential Oil in Sustainable Washing Treatments. Front. Sustain. Food Syst. 2020, 4, 1-9. [CrossRef]

44. Gębarowska, E.; Pytlarz-Kozicka, M.; Nöfer, J.; Łyczko, J.; Adamski, M.; Szumny, A. The Effect of Trichoderma spp. on the Composition of Volatile Secondary Metabolites and Biometric Parameters of Coriander (Coriandrum sativum L.). J. Food Qual. 2019, 2019. Article ID 5687032, 7p. [CrossRef]

45. Pellegrini, M.; Ricci, A.; Serio, A.; Chaves-López, C.; Mazzarrino, G.; D'Amato, S.; Lo Sterzo, C.; Paparella, A. Characterization of essential oils obtained from Abruzzo autochthonous plants: Antioxidant and antimicrobial activities assessment for food application. Foods 2018, 7, 19. [CrossRef]

46. Silva, F.; Ferreira, S.; Duarte, A.; Mendonca, D.I.; Domingues, F.C. Antifungal activity of Coriandrum sativum essential oil, its mode of action against Candida species and potential synergism with amphotericin B. Phytomedicine 2011, 19, 42-47. [CrossRef]

47. Rodrigues, V.H.; de Melo, M.M.R.; Portugal, I.; Silva, C.M. Extraction of Eucalyptus leaves using solvents of distinct polarity. Cluster analysis and extracts characterization. J. Supercrit. Fluids 2018, 135, 263-274. [CrossRef]

48. Vural, N.; Algan-Cavuldak, Ö.; Akay, M.A.; Anli, R.E. Determination of the various extraction solvent effects on polyphenolic profile and antioxidant activities of selected tea samples by chemometric approach. J. Food Meas. and Charact. 2020, 14, 1286-1305. [CrossRef]

49. Armatu, A.; Colceru-Mihul, S.; Bubueanu, C.; Draghici, E.; Pirvu, L. Evaluation of antioxidant and free scavenging potential of some Lamiaceae species growing in Romania. Rom. Biotechnol. Lett. 2010, 15, 5274-5280.

50. Chaouche, T.M.; Haddouchi, F.; Ksouri, R.; Medini, F.; El-Haci, I.A.; Boucherit, Z.; Sekkal, F.Z.; Atik-Bekara, F. Antioxidant Potential of Hydro-methanolic Extract of Prasium majus L: An in vitro Study. Pak. J. Biol. Sci. 2013, 16, 1318-1323. [CrossRef]

51. Ngo, Y.L.; Lau, C.H.; Chua, L.S. Review on rosmarinic acid extraction, fractionation and its anti-diabetic potential. Food Chem. Toxicol. 2018, 121, 687-700. [CrossRef] [PubMed]

52. Manzoor, M.F.; Ahmad, N.; Ahmed, Z.; Siddique, R.; Zeng, X.-A.; Rahaman, A.; Aadil, R.M.; Wahab, A. Novel extraction techniques and pharmaceutical activities of luteolin and its derivatives. J. Food Biochem. 2019, 43, e12974. [CrossRef] [PubMed]

53. Fierascu, R.C.; Fierascu, I.; Avramescu, S.M.; Sieniawska, E. Recovery of Natural Antioxidants from Agro-Industrial Side Streams through Advanced Extraction Techniques. Molecules 2019, 24, 4212. [CrossRef] [PubMed]

54. Maltese, F.; van der Kooy, F.; Verpoorte, R. Solvent Derived Artifacts in Natural Products Chemistry. Nat. Prod. Commun. 2009, 4, 447-454. [CrossRef]

55. Vaya, J.; Mahmood, S.; Goldblum, A.; Aviram, M.; Volkova, N.; Shaalan, A.; Musa, R.; Tamir, S. Inhibition of LDL oxidation by flavonoids in relation to their structure and calculated enthalpy. Phytochemistry 2003, 62, 89-99. [CrossRef]

56. Bazylko, A.; Strzelecka, H. A HPTLC densitometric determination of luteolin in Thymus vulgaris and its extracts. Fitoterapia 2007, 78, 391-395. [CrossRef]

57. Zengin, G.; Menghini, L.; di Sotto, A.; Mancinelli, R.; Sisto, F.; Carradori, S.; Cesa, S.; Fraschetti, C.; Filippi, A.; Angiolella, L. Chromatographic analyses, in vitro biological activities, and cytotoxicity of Cannabis sativa L. Essential oil: A multidisciplinary study. Molecules 2018, 23, 3266. [CrossRef]

58. Barros, L.; Duenas, M.; Dias, M.I.; Sousa, M.J.; Santos-Buelga, C.; Ferreira, I.C. Phenolic profiles of in vivo and in vitro grown Coriandrum sativum L. Food Chem. 2012, 132, 841-848. [CrossRef]

59. Msaada, K.; Jemia, M.B.; Salem, N.; Bachrouch, O.; Sriti, J.; Tammar, S.; Bettaieb, I.; Jabri, I.; Kefi, S.; Limam, F. Antioxidant activity of methanolic extracts from three coriander (Coriandrum sativum L.) fruit varieties. Arab. J. Chem. 2017, 10, CS3176-CS3183. [CrossRef]

60. Tohidi, B.; Rahimmalek, M.; Trindade, H. Review on essential oil, extracts composition, molecular and phytochemical properties of Thymus species in Iran. Ind. Crops Prod. 2019, 134, 89-99. [CrossRef]

61. Sena, S.R.; Dantas, T.R.; Pereira, C.G. Extracts from Thymus vulgaris and Origanum vulgare L. obtained by different separation processes: Global yield and functional profile. Trends Phytochem. Res. 2018, 2, 13-20. 
62. Sriti, J.; Wannes, W.A.; Talou, T.; Jemia, M.B.; Kchouk, M.E.; Marzouk, B. Antioxidant properties and polyphenol contents of different parts of coriander (Coriandrum sativum L.) fruit. La Rivista Italiana Delle Sostanze Grasse 2012, 49, 253-262.

63. Moccia, S.; Siano, F.; Russo, G.L.; Volpe, M.G.; La Cara, F.; Pacifico, S.; Piccolella, S.; Picariello, G. Antiproliferative and antioxidant effect of polar hemp extracts (Cannabis sativa L., Fedora cv.) in human colorectal cell lines. Int. J. Food Sci. Nutr. 2020, 71, 410-423. [CrossRef] [PubMed]

64. Smeriglio, A.; Galati, E.M.; Monforte, M.T.; Lanuzza, F.; D’Angelo, V.; Circosta, C. Polyphenolic Compounds and Antioxidant Activity of Cold-Pressed Seed Oil from Finola Cultivar of Cannabis sativa L. Phytother. Res. 2016, 30, 1298-1307. [CrossRef]

65. Hsu, B.; Coupar, I.M.; Ng, K. Antioxidant activity of hot water extract from the fruit of the Doum palm, Hyphaene Thebaica. Food Chem. 2006, 98, 317-328. [CrossRef]

66. Van der Sluis, A.A.; Dekker, M.; van Boekel, M.A.J.S. Activity and concentration of polyphenolic antioxidants in apple juice. 3. Stability during storage. J. Agric. Food Chem. 2005, 53, 1073-1080. [CrossRef]

67. Cheng, Z.; Su, L.; Moore, J.; Zhou, K.; Luther, M.; Yin, J.-J.; Yu, L. Effects of postharvest treatment and heat stress on availability of wheat antioxidants. J. Agric. Food Chem. 2006, 54, 5623-5629. [CrossRef]

68. Dutra, R.; Leite, M.; Barbosa, N. Quantification of phenolic constituents and antioxidant activity of Pterodon Emarginatus vogel seeds. Int. J. Mol. Sci. 2008, 9, 606. [CrossRef]

69. Antolovich, M.; Prenzler, P.; Robards, K.; Ryan, D. Sample preparation in the determination of phenolic compounds in fruits. Analyst 2000, 125, 989-1009. [CrossRef]

(C) 2020 by the authors. Licensee MDPI, Basel, Switzerland. This article is an open access article distributed under the terms and conditions of the Creative Commons Attribution (CC BY) license (http://creativecommons.org/licenses/by/4.0/). 